\title{
ON CONVERGENCE OF SEMI-DISCRETE HIGH RESOLUTION SCHEMES WITH VAN LEER'S FLUX LIMITER FOR CONSERVATION LAWS*
}

\author{
NAN JIANG ${ }^{\dagger}$ AND HUANAN YANG ${ }^{\ddagger}$
}

\begin{abstract}
In the early 1980s, Sweby [19] investigated a class of high resolution schemes using flux limiters for hyperbolic conservation laws. For the convex homogeneous conservation laws, Yang [23] has shown the convergence of the numerical solutions of semi-discrete schemes based on minmod limiter when the general building block of the schemes is an arbitrary $E$-scheme, and based on Chakravarthy-Osher limiter when the building block of the schemes is the Godunov, the EngquistOsher, or the Lax-Friedrichs to the physically correct solution. Recently, Yang and Jiang [25] have proved the convergence of these schemes for convex conservation laws with a source term. However, the convergence problems of other flux limiter, such as van Leer and superbee have been open. In this paper, we apply the convergence criteria, established in [23] [25] by using Yang's wavewise entropy inequality (WEI) concept, to prove the convergence of the semi-discrete schemes with van Leer's limiter for the aforementioned three building blocks. The result is valid for scalar convex conservation laws in one space dimension with or without a source term. Thus, we have settled one of the aforementioned problems.
\end{abstract}

Key words. Conservation law with source terms, schemes with flux limiters, entropy condition, convergence

AMS subject classifications. 65M12(35L60)

1. Introduction. The goal of this paper is to prove the convergence to the the entropy solution of the semi-discrete high resolution schemes based on van Leer's famous flux limiter [21] for the initial value problems of hyperbolic conservation laws with source terms:

$$
\left\{\begin{array}{l}
w_{t}+f(w)_{x}=q(w) \\
w(x, 0)=w_{0}(x)
\end{array}\right.
$$

where $f \in C^{1}(\mathbb{R})$ is convex, $q \in C^{1}(\mathbb{R})$, and $w_{0} \in B V(\mathbb{R})$. Here $B V$ stands for the subspace of $L_{l o c}^{1}$ consisting of functions $z$ with bounded total variation

$$
T V(z):=\sup _{h \neq 0} \int_{\mathbb{R}} \frac{|z(x+h)-z(x)|}{|h|} d x .
$$

The corresponding homogeneous problems to (1.1) are

$$
\left\{\begin{array}{l}
w_{t}+f(w)_{x}=0 \\
w(x, 0)=w_{0}(x)
\end{array}\right.
$$

It is well known that the solution of (1.1) may develop discontinuities in finite time. Therefore, we seek weak solutions $w$ that satisfies:

$$
\int_{\mathbb{R}} \int_{0}^{T}\left(w \phi_{t}+f(w) \phi_{x}+q(w) \phi\right) d x d t=-\int_{\mathbb{R}} w_{0}(x) \phi(x, 0) d x
$$

\footnotetext{
*Received June 25, 2005; accepted for publication October 28, 2005.

${ }^{\dagger}$ Department of Mathematical Sciences, University of South Dakota, Vermillion, SD 57069, USA (njiang@usd.edu).

${ }_{\ddagger}^{\ddagger}$ Department of Mathematics, Kansas State University, Manhattan, KS 66506, USA (hyang@ math.ksu.edu).
} 
for all $\phi \in C_{0}^{1}(\mathbb{R} \times[0, T))$. The week solutions are not necessarily unique and the entropy conditions are required to single out the physically relevant one, which, for all convex entropy function $V$ and its flux $F$, i.e. $V^{\prime}(w) f^{\prime}(w)=F^{\prime}(w)$, satisfies the inequality [10] [22]

$$
V(w)_{t}+F(w)_{x} \leq V^{\prime}(w) q(w)
$$

in the sense of distribution. Since $f$ is convex, the entropy inequality (1.5) for the entropy function $V(w)=w^{2}$ is sufficient to ensure the uniqueness of the solution [3].

The semi-discrete high resolution schemes we consider have the form

$$
\frac{d u_{j}(t)}{d t}=-\frac{1}{h}\left(g_{j+\frac{1}{2}}(t)-g_{j-\frac{1}{2}}(t)\right)+q\left(u_{j}(t)\right),
$$

where the numerical flux $g$ is given by

$$
g_{j+\frac{1}{2}}(t)=g\left(u_{j-p+1}(t), u_{j-p+2}(t), \cdots, u_{j}(t), \cdots, u_{j+p}(t), h\right),
$$

which is Lipschitz continuous with respect to its first $2 p$ arguments and is consistent with the conservation law in the sense that

$$
g(u, u, \cdots, u, h) \equiv f(u) .
$$

The scheme (1.6) is said to be self-similar if $g$ is independent of $h$. In this paper, we only consider numerical flux $g$ that is self-similar. It should be pointed out that it is in the case of self-similar schemes that one faces the most formidable challenge of entropy analysis. See [23] for a comment on entropy analysis of schemes with step-size dependant fluxes.

When $q \equiv 0$ in (1.6), that is

$$
\frac{d u_{j}(t)}{d t}=-\frac{1}{h}\left(g_{j+\frac{1}{2}}(t)-g_{j-\frac{1}{2}}(t)\right)
$$

we call the corresponding schemes (1.7)-(1.9) the homogeneous counterpart (HCP) of (1.6)-(1.8) that approximate problems (1.3).

A scheme (1.6)-(1.8) for the Cauchy problem (1.1) converges if, for every initial condition $w_{0}$ in $\mathrm{BV}$ and for each sequence of initial data $\left\{u_{j}^{k}(0), j \in \mathbb{Z}\right\}_{k=1}^{\infty}$ with uniformly bounded variations that converges in $L_{l o c}^{1}(\mathbb{R})$ to $w_{0}$, the corresponding sequence of (extended) numerical solutions $\left\{u^{k}\right\}$ generated by the scheme converges in $L_{l o c}^{1}(\mathbb{R} \times[0, T))$ to the unique entropy solution $w$ of the problem (1.1) provided that the step sizes $h_{k}$ of $u^{k}$ vanish as $k \rightarrow \infty$.

Together with MUSCL scheme, flux limiter method was pioneered by van Leer in 1970s, aimed at combining the merits of high accuracy of classical second order schemes such as Lax-Wendroff and Beam-Warming schemes [11] and noise(spurious oscillations)-free shock profiles of first order monotone schemes such as Godunov [5], Lax-Friedrichs [12], or Engquist-Osher's [4] schemes. In 1984, guided by Harten's theory of high resolution schemes, Sweby [19] cast several flux limiter methods including van Leer [21], Roe [17], and Chakravarthy and Osher's methods [1] into a unified framework, in which the flux limiter is a function $\psi(r)$ where $r$ measures the smoothness of the solution: $r \approx 1$ where the numerical solution is smooth, $r$ is away from one near the discontinuity and $r \leq 0$ at the spatial extrema. Roughly speaking, in constructing of the numerical flux, $\psi(r)$ is one of the two factors forming the 
anti-diffusion added to the flux of a monotone scheme, or more generally a $E$-scheme, which we called the building block of the scheme; the other factor is essentially the difference of the flux of a second order central difference scheme and that of the building block. To accommodate the upwinding principle, the addition of the anti-diffusion is split into a backward difference term and a forward difference term. Sweby noticed that if $\psi$ is Lipschitz continuous and its graph lies in the region defined by

$$
\left\{\left(r, \psi_{\Phi}(r)\right): \psi_{\Phi}(r)=\max (0, \min (\Phi r, 1), \min (r, \Phi)), 1 \leq \Phi \leq 2, r \in \mathbb{R}\right\},
$$

then the scheme is second order accurate away from discontinuity and spatial extrema, and is TVD (total variation diminishing), and hence, is qualified to be a high resolution scheme in the sense of Harten [7].

The successes of this class of schemes made it one of the two pillars (the other is the MUSCL method) in the early stage of the great campaign for development of high resolution shock capturing schemes, which has spanned about three decades since 1970s and has produced, among others, such powerful schemes as PPM schemes [2], ENO schemes [6] [8] [18] and WENO schemes [13] [9]. This campaign is without doubt one of the most successful stories of modern numerical analysis and computational fluid dynamics.

Contrary to the computational successes, theoretical analysis of the flux limiter methods has been lagged far behind. By the beginning of 1990s, there had been no rigorous result answering the critical question of whether the numerical solutions of the methods converge to the unique entropy solution, except that Osher [15] and Osher and Tadmor [16] gave positive answers for some flux limiter methods modified or designed to meet certain cell entropy inequalities. Though elegant, the method of cell entropy inequality (CEL) demands too much, and can not be applied to settle the convergence of the flux limiter methods in their classical form, say, described by Sweby [19], even for the semi-discrete version.

A significant progress was made in the 1990s when Yang [23] introduced the concept of wavewise entropy inequality (WEI). Based on this concept, a series of criteria of convergence was established. For convex conservation laws, one of Yang's criteria points out that, a wavewise entropy inequality across the area of rarefaction where $u_{j} \leq u_{j+1}$ for all $x_{j}$ is sufficient for convergence to the entropy solution. Hence, in convergence analysis, one may safely remove the shock area from scrutiny. Further, even in the rarefaction area, a much weaker condition than CEI is sufficient for convergence. Using this criterion, Yang proved convergence of semi-discrete high resolution schemes based on the minmod flux limiter with an arbitrary $E$-flux building block, or based on Chakravarthy-Osher flux limiter with Lax-Friedrichs, Godunov, or EngquistOsher building block. We would mention that Yang also established convergence of semi-discrete generalized MUSCL scheme [23] and of explicit MUSCL scheme for a CFL number up to 0.5 [24].

Recently, for the semi-discrete case, Yang and Jiang [25] have extended Yang's entire WEI framework to the non-homogeneous conservation laws provided that the numerical flux satisfies the same conditions as in the homogeneous case. Surprisingly, without any change, the aforementioned convergence criterion originally developed for homogeneous conservation laws is also valid for convex conservation laws with an arbitrary $C^{1}$ source term. Hence, we were able to establish convergence of all the aforementioned semi-discrete schemes for convex conservation laws with an arbitrary $C^{1}$ source term.

In this paper, we will use the WEI convergence criterion, formulated in [25], to show the entropy consistency of the schemes using van Leer's flux limiter when the 
building block of the schemes is the Lax-Friedrichs scheme, or a class of $E$-schemes of which the Godunov and the Engquist-Osher schemes are special cases. The results obtained here will be valid for convex conservation laws with or without a source term. The paper is organized as follows. In section 2, we present the main result of this paper. In section 3 , we give the proof of the main result.

2. The main result. In [25], we have shown that the following separation property is a sufficient condition for the schemes (1.6)-(1.8) to be TVB (total variation bounded) schemes. This property was first derived by Tadmor [20] as a convenient TVD condition for the schemes (1.7)-(1.9).

Assumption 2.1. The numerical fluxes $g_{j+\frac{1}{2}}(t), j=0, \pm 1, \pm 2, \cdots$, satisfy

$$
g_{j+\frac{1}{2}}(t) \geq f\left(u_{j}\right) \geq g_{j-\frac{1}{2}}(t) \quad \text { if } u_{j}(t)-u_{j \pm 1}(t) \geq 0,
$$

and

$$
g_{j+\frac{1}{2}}(t) \leq f\left(u_{j}\right) \leq g_{j-\frac{1}{2}}(t) \quad \text { if } u_{j}(t)-u_{j \pm 1}(t) \leq 0 .
$$

We also have shown that the schemes satisfying the above assumption are extremum traceable schemes. Therefore the WEI convergence criteria, established in [25], are applicable. Let $f[w ; L, R]$ be the linear function interpolating of $f(w)$ at $w=L$ and $w=R$. For a convex conservation law, a pair of numbers $\{L, R\}$ is called a rarefying pair if $L<R$ and $f[w ; L, R]>f(w)$ when $L<w<R$. And a collection of data $\left\{v_{j}\right\}_{j=-p}^{n+p}$ is called a rarefying collection with respect to the pair $\{L, R\}$ if $L=v_{0} \leq v_{1} \leq \cdots \leq v_{n}=R$, and $L \leq v_{-1}$, and $R \geq v_{n+1}$. Let $\bar{g}_{j+1 / 2}:=g\left(v_{j-p+1}, v_{j-p+2}, \cdots, v_{j+p}\right)$, where $g$ is the function (1.7) in its self-similar form. We are now ready to quote the following all-powerful WEI convergence criterion which was first established in [23] for homogeneous conservation laws and was extended in [25] for conservation laws with an arbitrary $C^{1}$ source term.

TheOREm 2.2. A scheme of the form (1.6)-(1.8) satisfying Assumption 2.1 converges for convex conservation laws (1.1) if, for any rarefying pair $\{L, R\}$, there is a constant $\delta>0$ such that the quadrature type inequality

$$
\sum_{j=0}^{n-1}\left(v_{j+1}-v_{j}\right) \bar{g}_{j+1 / 2}+\delta<\int_{L}^{R} f[w ; L, R] d w
$$

holds for all rarefying collections $\left\{v_{j}\right\}_{j=-p}^{n+p}$ with respect to the pair $\{L, R\}$.

Following Osher and Chakravarthy [15], we now discuss the semi-discrete version of the high resolution schemes with flux limiters in the framework of Sweby [19]. Let $g^{E}(\cdot, \cdot)$ be the flux of any $E$-scheme (see Osher [14]), i.e., it is Lipschitz continuous, and, for all $w$ between $w_{j}$ and $w_{j+1}$, it satisfies

$$
\operatorname{sgn}\left(w_{j+1}-w_{j}\right)\left(g^{E}\left(w_{j}, w_{j+1}\right)-f(w)\right) \leq 0 .
$$

We borrow the following notations from [19]. Denote

$$
\left(\Delta f_{j+\frac{1}{2}}\right)^{+}:=f\left(u_{j+1}\right)-g^{E}\left(u_{j}, u_{j+1}\right),
$$


and

$$
\left(\Delta f_{j+\frac{1}{2}}\right)^{-}:=f\left(u_{j}\right)-g^{E}\left(u_{j}, u_{j+1}\right) .
$$

We set

$$
r_{j}^{+}:=\left(\Delta f_{j-\frac{1}{2}}\right)^{+} /\left(\Delta f_{j+\frac{1}{2}}\right)^{+}, \quad r_{j}^{-}:=\left(\Delta f_{j+\frac{1}{2}}\right)^{-} /\left(\Delta f_{j-\frac{1}{2}}\right)^{-},
$$

and

$$
\left(D f_{j+\frac{1}{2}}\right)^{ \pm}:=\left(\Delta f_{j+\frac{1}{2}}\right)^{ \pm} / \Delta u_{j+\frac{1}{2}}
$$

Let $\psi$ be a flux limiter function. Using notations (2.3)-(2.5), the numerical flux with the limiter $\psi$ is defined by

$$
g_{j+\frac{1}{2}}=g^{E}\left(u_{j}, u_{j+1}\right)+\frac{1}{2} \psi\left(r_{j}^{+}\right)\left(\Delta f_{j+\frac{1}{2}}\right)^{+}+\frac{1}{2} \psi\left(r_{j+1}^{-}\right)\left(\Delta f_{j+\frac{1}{2}}\right)^{-},
$$

where $g^{E}$ is the numerical flux function of any $E$-scheme.

To facilitate the readers, we now quote the definitions of the numerical fluxes of the aforementioned three monotone schemes.

The Godunov scheme (see [5]):

$$
g^{\operatorname{God}}\left(u_{j}, u_{j+1}\right)=\left\{\begin{array}{lll}
\min _{u_{j} \leq w \leq u_{j+1}} f(w) & \text { when } & u_{j} \leq u_{j+1} \\
\max _{u_{j} \geq w \geq u_{j+1}} f(w) & \text { when } & u_{j} \geq u_{j+1}
\end{array}\right.
$$

The Engquist-Osher scheme (see [4]):

$$
g^{E O}\left(u_{j}, u_{j+1}\right)=\int_{0}^{u_{j}} \max \left(f^{\prime}(w), 0\right) d w+\int_{0}^{u_{j+1}} \min \left(f^{\prime}(w), 0\right) d w+f(0) .
$$

The Lax-Friedrichs scheme (see [12]):

$$
g^{L F}\left(u_{j}, u_{j+1}\right)=\frac{f\left(u_{j}\right)+f\left(u_{j+1}\right)}{2}-\frac{a}{2}\left(u_{j+1}-u_{j}\right)
$$

where $a \geq \max \left|f^{\prime}(w)\right|$.

Let $s$ be a sonic point: $f^{\prime}(s)=0$. The Godunov and Engquist-Osher schemes belong to a class of $E$-schemes that satisfies

$$
g^{E}(x, y)= \begin{cases}f(x) & \text { if } s \leq x \leq y \\ f(y) & \text { if } x \leq y \leq s\end{cases}
$$

and

$$
\begin{aligned}
& g^{E}(x, y) \geq \\
& \max \left\{f(y)-4 f^{\prime}(y)(y-x), f(x)+4 f^{\prime}(x)(y-x)\right\} \quad \text { if } x \leq s \leq y .
\end{aligned}
$$

The main result of this paper concerning the convergence to the entropy solution of the semi-discrete high resolution schemes based on van Leer's flux limiter:

$$
\psi_{V L}(r)= \begin{cases}0 & r \leq 0 \\ \frac{2 r}{1+r} & r>0\end{cases}
$$


when the building block of the schemes is either the Lax-Friedrichs scheme, or the aforementioned class of $E$-schemes. The main result of this paper is the following.

THEOREM 2.3. The numerical solutions of the schemes (1.6)-(1.8) ((1.7)(1.9)resp.), for the convex problems (1.1) ((1.3) resp.), converge provided that the numerical flux $g_{j+\frac{1}{2}}$ is defined by (2.7), where $\psi=\psi_{V L}$ is the van Leer flux limiter given by (2.13), and $g^{E}(\cdot, \cdot)$ is either the numerical flux of the Lax-Friedrichs scheme or that of one of the class of E-schemes of which the numerical flux satisfies (2.11) and (2.12).

REMARK 1. Since the numerical flux (2.7) is an increasing affine functional of the limiter $\psi$, the following hold: 1 . if two schemes with the flux limiters $\psi_{1}$ and $\psi_{2}$ respectively both satisfy the WEI convergence criterion of Theorem 2.2, and $c_{1}$ and $c_{2}$ are two nonnegative constants with $c_{1}+c_{2}=1$, then the scheme with the flux limiter $\psi=c_{1} \psi_{1}+c_{2} \psi_{2}$ also converges. 2. if $\psi_{1}(r) \leq \psi_{2}(r)$ for $r \geq 0$, and the scheme with the flux limiter $\psi_{2}$ satisfies the WEI convergence criterion of Theorem 2.2, then, the scheme with the flux limiter $\psi_{1}$ also converges.

REMARK 2. We can significantly strengthen the theorem by weakening the conditions of the theorem since the WEI criterion requires nothing at shock area, provided that the Assumption 2.1 holds. Hence, the high resolution schemes still converge if the conditions concerning the van Leer flux limiter are replaced by the following much weaker ones:

(i) The inequality $g_{j+\frac{1}{2}} \geq f\left(u_{j}\right)$ holds if $u_{j} \geq \max \left(u_{j-1}, u_{j+1}\right)$, and $g_{j-\frac{1}{2}} \geq f\left(u_{j}\right)$ holds if $u_{j} \leq \min \left(u_{j-1}, u_{j+1}\right)$.

(ii) When $u_{j+1}>u_{j}, g_{j+\frac{1}{2}}$ is defined by (2.7), where $\psi=\psi_{V L}$ is the van Leer flux limiter given by (2.13). The following proof is for the thus strengthened Theorem 2.3.

\section{Proof of the main result.}

Proof. [Proof of Theorem 2.3] We first show that the scheme satisfies the Assumption 2.1. Let $u_{j+1}$ be a spatial maximum; thus $r_{j+1}^{-} \leq 0$. Hence, it follows from (2.13) that $\psi\left(r_{j+1}^{-}\right)=0$. Therefore

$$
\begin{aligned}
g_{j+\frac{1}{2}} & =g^{E}\left(u_{j}, u_{j+1}\right)+\frac{1}{2} \psi\left(r_{j}^{+}\right)\left(\Delta f_{j+\frac{1}{2}}\right)^{+} \\
& \leq g^{E}\left(u_{j}, u_{j+1}\right)+\left(\Delta f_{j+\frac{1}{2}}\right)^{+} \\
& =f\left(u_{j+1}\right) .
\end{aligned}
$$

Replacing $j$ with $j-1$ in the preceding inequality, we see that if $u_{j}-u_{j \pm 1} \geq 0$, then $g_{j-\frac{1}{2}} \leq f\left(u_{j}\right)$. Similarly, if $u_{j}-u_{j \pm 1} \leq 0$, then $g_{j+\frac{1}{2}} \leq f\left(u_{j}\right)$. These two inequalities and condition (i) verify Assumption 2.1.

Next, let $\{L, R\}$ be an arbitrary rarefying pair $\left\{v_{j}\right\}_{j=-p}^{n+p}$ and $\bar{g}_{j+\frac{1}{2}}$ be as in Theorem 2.2. We keep the same notations $\left(\Delta f_{j+\frac{1}{2}}\right)^{ \pm}, r_{j}^{ \pm}$, and $\left(D f_{j+\frac{1}{2}}\right)^{ \pm}$for $\left\{v_{j}\right\}$ instead of $\left\{u_{j}\right\}$ here. We also use the following notation for the divided difference:

$$
f_{j+\frac{1}{2}}^{\prime}:=\frac{f\left(v_{j+1}\right)-f\left(v_{j}\right)}{v_{j+1}-v_{j}} .
$$


We now claim that the inequality

$$
\sum_{j=0}^{n-1}\left(S_{j+\frac{1}{2}}-\bar{f}\left[v_{j}, v_{j+1}\right]\right) \leq 0
$$

holds, where

$$
\bar{f}[c, d]:=\int_{c}^{d}\{f[w ; c, d]-f(w)\} d w
$$

for $c<d$, and

$$
S_{j+\frac{1}{2}}:=\int_{v_{j}}^{v_{j+1}}\left[\bar{g}_{j+\frac{1}{2}}-f(w)\right] d w .
$$

Equipped by inequality (3.2), we are able to prove the theorem. Indeed, according to the Theorem 2.2, to prove the convergence of the scheme, it suffices to show that for any rarefying pair $\{L, R\}$, there exists a $\delta>0$ such that for any rarefying collection $\left\{v_{j}\right\}_{j=-p}^{n+p}$ with respect to the pair, the inequality (2.1) holds. Using (3.3) and (3.4), we rewrite the inequality (2.1) as

$$
\sum_{j=0}^{n-1} S_{j+\frac{1}{2}}<\bar{f}[L, R]-\delta
$$

Now, by the convexity of $f$, the following inequality holds

$$
\bar{f}[x, y]+\bar{f}[y, z] \leq \bar{f}[x, z] \text { for } \quad x \leq y \leq z .
$$

This and (3.2) yield

$$
\sum_{j=0}^{n-1} S_{j+\frac{1}{2}} \leq \sum_{j=0}^{n-1} \bar{f}\left[v_{j}, v_{j+1}\right] \leq \bar{f}\left[L, v_{i}\right]+\bar{f}\left[v_{i}, R\right] \leq \bar{f}[L, R] .
$$

Assume that for each $\delta>0$ there exists a rarefying collection $\left\{v_{j}\right\}_{j=-p}^{n+p}$, with respect to the pair $\{L, R\}$ for some positive integer $n$, violates (3.5). Then (3.7) implies that there exists a sequence of rarefying collections $\left\{v_{j}^{\nu}, j=-p,-p+1, \cdots, n^{\nu}+p\right\}_{\nu=1}^{\infty}$ with respect to the pair $\{L, R\}$ such that

$$
\lim _{\nu \rightarrow \infty} \sum_{j=0}^{n^{\nu}-1} S_{j+\frac{1}{2}}^{\nu}=\lim _{\nu \rightarrow \infty} \sum_{j=0}^{n^{\nu}-1} \bar{f}\left[v_{j}^{\nu}, v_{j+1}^{\nu}\right]=\bar{f}[L, R]
$$

This implies that there exists a sequence of integers $\left\{j^{\nu}\right\}$ satisfying $0 \leq j^{\nu} \leq n^{\nu}-1$ such that

$$
\lim _{\nu \rightarrow \infty} v_{j^{\nu}}^{\nu}=L \quad \text { and } \quad \lim _{\nu \rightarrow \infty} v_{j^{\nu}+1}^{\nu}=R
$$

For, otherwise, there would exist a constant $\rho>0$, a subsequence of the rarefying collections, still denoted by $\left\{v_{j}^{\nu}, j=-p,-p+1, \cdots, n^{\nu}+p\right\}_{\nu=1}^{\infty}$, and a sequence of integers $\left\{i^{\nu}\right\}$ satisfying $1 \leq i^{\nu} \leq n^{\nu}-1$ such that 
$L+\rho<v_{i^{\nu}}^{\nu}<R-\rho$. Then the convexity of $f$, the definition of rarefying pairs, and the inequality (3.7) would imply

$$
\begin{aligned}
& \sum_{j=0}^{n^{\nu}-1} \bar{f}\left[v_{j}^{\nu}, v_{j+1}^{\nu}\right] \\
\leq & \bar{f}\left[L, v_{i^{\nu}}^{\nu}\right]+\bar{f}\left[v_{i^{\nu}}^{\nu}, R\right] \\
\leq & H_{\rho}:=\max (\bar{f}[L, L+\rho]+\bar{f}[L+\rho, R], \bar{f}[L, R-\rho]+\bar{f}[R-\rho, R]) \\
< & \bar{f}[L, R] .
\end{aligned}
$$

This would contradict (3.8) because $H_{\rho}$ is independent of $\nu$. Hence, we have confirmed the existence of a sequence $\left\{j^{\nu}\right\}$ satisfying (3.9). Now, combining (3.6), (3.7), (3.8) and (3.9), we obtain

$$
\begin{aligned}
\bar{f}[L, R] & =\lim _{\nu \rightarrow \infty} \sum_{j=0}^{n^{\nu}-1} S_{j+\frac{1}{2}}^{\nu} \\
& =\lim _{\nu \rightarrow \infty}\left(\sum_{j=0}^{j^{\nu}-1} S_{j+\frac{1}{2}}^{\nu}+S_{j^{\nu}+\frac{1}{2}}^{\nu}+\sum_{j=j^{\nu}+1}^{n^{\nu}-1} S_{j+\frac{1}{2}}^{\nu}\right) \\
& \leq \lim _{\nu \rightarrow \infty}\left(\bar{f}\left[L, v_{j^{\nu}}^{\nu}\right]+S_{j^{\nu}+\frac{1}{2}}^{\nu}+\bar{f}\left[v_{j^{\nu}+1}^{\nu}, R\right]\right) \\
& =\lim _{\nu \rightarrow \infty} S_{j^{\nu}+\frac{1}{2}}^{\nu} \\
& \leq \bar{f}[L, R] .
\end{aligned}
$$

This implies

$$
\lim _{\nu \rightarrow \infty} S_{j^{\nu}+\frac{1}{2}}^{\nu}=\bar{f}[L, R] .
$$

However, applying (3.9) and the definition of the rarefying collections with respect to the rarefying pair $\{L, R\}$ to $(2.7)$, we get

$$
\lim _{\nu \rightarrow \infty} \bar{g}_{j^{\nu}+\frac{1}{2}}^{\nu}=g^{E}(L, R)
$$

and hence, by (3.4),

$$
\lim _{\nu \rightarrow \infty} S_{j^{\nu}+\frac{1}{2}}^{\nu} \leq 0
$$

This contradicts (3.12) since $\bar{f}[L, R]>0$ by the definition of the rarefying pair $\{L, R\}$.

It remains to justify the inequality (3.2). Since $\psi(r)=\psi_{V L}(r)=\frac{2 r}{1+r}$ for $r \geq 0$, the numerical flux (2.7), evaluated at a rarefying collection $\left\{v_{j}\right\}_{j=-p}^{n+p}$ with respect to any rarefying $\{L, R\}$, can be written as:

$$
\bar{g}_{j+\frac{1}{2}}=g_{j+\frac{1}{2}}^{E}+\frac{\left(\Delta f_{j-\frac{1}{2}}\right)^{+}\left(\Delta f_{j+\frac{1}{2}}\right)^{+}}{\left(\Delta f_{j-\frac{1}{2}}\right)^{+}+\left(\Delta f_{j+\frac{1}{2}}\right)^{+}}+\frac{\left(\Delta f_{j+\frac{1}{2}}\right)^{-}\left(\Delta f_{j+\frac{3}{2}}\right)^{-}}{\left(\Delta f_{j+\frac{1}{2}}\right)^{-}+\left(\Delta f_{j+\frac{3}{2}}\right)^{-}} .
$$

Now, by (2.3) and (2.4), we have

$$
\int_{v_{j}}^{v_{j+1}}\left[g_{j+\frac{1}{2}}^{E}-\frac{f\left(v_{j+1}\right)+f\left(v_{j}\right)}{2}\right] d w=-\frac{\left(\Delta f_{j+\frac{1}{2}}\right)^{-}+\left(\Delta f_{j+\frac{1}{2}}\right)^{+}}{2} \Delta v_{j+\frac{1}{2}} .
$$


Therefore,

$$
\sum_{j=0}^{n-1}\left(S_{j+\frac{1}{2}}-\bar{f}\left[v_{j}, v_{j+1}\right]\right)=\frac{1}{2}\left(P_{1}+P_{2}\right)
$$

where

$$
P_{1}:=\sum_{j=0}^{n-1} \frac{\left(\Delta f_{j-\frac{1}{2}}\right)^{+}-\left(\Delta f_{j+\frac{1}{2}}\right)^{+}}{\left(\Delta f_{j-\frac{1}{2}}\right)^{+}+\left(\Delta f_{j+\frac{1}{2}}\right)^{+}}\left(\Delta f_{j+\frac{1}{2}}\right)^{+} \Delta v_{j+\frac{1}{2}},
$$

and

$$
P_{2}:=\sum_{j=0}^{n-1} \frac{\left(\Delta f_{j+\frac{3}{2}}\right)^{-}-\left(\Delta f_{j+\frac{1}{2}}\right)^{-}}{\left(\Delta f_{j+\frac{3}{2}}\right)^{-}+\left(\Delta f_{j+\frac{1}{2}}\right)^{-}}\left(\Delta f_{j+\frac{1}{2}}\right)^{-} \Delta v_{j+\frac{1}{2}} .
$$

In this last part of the proof, the argument for the Lax-Friedrichs building block are different from that for the class of $E$-schemes of which the numerical flux satisfies (2.11) and (2.12). In both cases the following elementary inequality is helpful:

$$
\frac{\alpha-\beta}{\alpha+\beta} \beta \leq \frac{\alpha-\beta}{2} \quad \forall \alpha, \beta \in \mathbb{R} \quad \text { with } \quad \alpha+\beta>0 .
$$

Let us first deal with the case of Lax-Friedrichs flux. Applying the inequality (3.14) to $P_{1}$ and $P_{2}$, we have

$$
\begin{aligned}
& \sum_{j=0}^{n-1}\left(S_{j+\frac{1}{2}}-\bar{f}\left[v_{j}, v_{j+1}\right]\right) \\
\leq & \frac{1}{4} \sum_{j=0}^{n-1}\left[\left(\Delta f_{j-\frac{1}{2}}\right)^{+}-\left(\Delta f_{j+\frac{1}{2}}\right)^{+}+\left(\Delta f_{j+\frac{3}{2}}\right)^{-}-\left(\Delta f_{j+\frac{1}{2}}\right)^{-}\right] \Delta v_{j+\frac{1}{2}} .
\end{aligned}
$$

Noticing that (2.6) and (3.1) implies the equality

$$
\left(D f_{j+\frac{1}{2}}\right)^{ \pm}=\left(a \pm f_{j+\frac{1}{2}}^{\prime}\right) / 2
$$

we have

$$
\begin{aligned}
& \sum_{j=0}^{n-1}\left[\left(\Delta f_{j-\frac{1}{2}}\right)^{+}-\left(\Delta f_{j+\frac{1}{2}}\right)^{+}+\left(\Delta f_{j+\frac{3}{2}}\right)^{-}-\left(\Delta f_{j+\frac{1}{2}}\right)^{-}\right] \Delta v_{j+\frac{1}{2}} \\
= & -\sum_{j=0}^{n-1}\left[\left(\Delta f_{j+\frac{1}{2}}\right)^{+}+\left(\Delta f_{j+\frac{1}{2}}\right)^{-}\right] \Delta v_{j+\frac{1}{2}} \\
+ & \sum_{j=0}^{n-1}\left[\left(\Delta f_{j-\frac{1}{2}}\right)^{+}+\left(\Delta f_{j+\frac{3}{2}}\right)^{-}\right] \Delta v_{j+\frac{1}{2}} \\
= & -\sum_{j=0}^{n-1}\left[\left(D f_{j+\frac{1}{2}}\right)^{+}+\left(D f_{j+\frac{1}{2}}\right)^{-}\right]\left(\Delta v_{j+\frac{1}{2}}\right)^{2} \\
+ & \sum_{j=0}^{n-1}\left[\left(D f_{j-\frac{1}{2}}\right)^{+} \Delta v_{j-\frac{1}{2}} \Delta v_{j+\frac{1}{2}}+\left(D f_{j+\frac{3}{2}}\right)^{-} \Delta v_{j+\frac{1}{2}} \Delta v_{j+\frac{3}{2}}\right] \\
= & -a\left(\Delta v_{0+\frac{1}{2}}\right)^{2}+\frac{a}{2} \Delta v_{0-\frac{1}{2}} \Delta v_{0+\frac{1}{2}}+\frac{a}{2} \Delta v_{n-\frac{1}{2}} \Delta v_{n+\frac{1}{2}}+\frac{1}{2} E+C,
\end{aligned}
$$


where

$$
E:=\sum_{j=0}^{n-1}\left(f_{j-\frac{1}{2}}^{\prime} \Delta v_{j-\frac{1}{2}} \Delta v_{j+\frac{1}{2}}-f_{j+\frac{3}{2}}^{\prime} \Delta v_{j+\frac{1}{2}} \Delta v_{j+\frac{3}{2}}\right)
$$

and

$$
C:=\sum_{j=1}^{n-1}\left[-a\left(\Delta v_{j+\frac{1}{2}}\right)^{2}+a \Delta v_{j-\frac{1}{2}} \Delta v_{j+\frac{1}{2}}\right] .
$$

First, the convexity of $f$ implies that $f_{j+\frac{1}{2}}^{\prime} \leq f_{j+\frac{3}{2}}^{\prime}$ holds for $0 \leq j \leq n-2$, which ensures that

$$
E \leq f_{0-\frac{1}{2}}^{\prime} \Delta v_{0-\frac{1}{2}} \Delta v_{0+\frac{1}{2}}-f_{n+\frac{1}{2}}^{\prime} \Delta v_{n-\frac{1}{2}} \Delta v_{n+\frac{1}{2}} .
$$

Next, it is easy to see that

$$
C \leq-\frac{1}{2} a\left(\Delta v_{n-\frac{1}{2}}\right)^{2}+\frac{1}{2} a\left(\Delta v_{0+\frac{1}{2}}\right)^{2} .
$$

Therefore,

$$
\begin{aligned}
& \sum_{j=0}^{n-1}\left[\left(\Delta f_{j-\frac{1}{2}}\right)^{+}-\left(\Delta f_{j+\frac{1}{2}}\right)^{+}+\left(\Delta f_{j+\frac{3}{2}}\right)^{-}-\left(\Delta f_{j+\frac{1}{2}}\right)^{-}\right] \Delta v_{j+\frac{1}{2}} \\
\leq & -a\left(\Delta v_{0+\frac{1}{2}}\right)^{2}+\frac{a}{2} \Delta v_{0-\frac{1}{2}} \Delta v_{0+\frac{1}{2}}+\frac{a}{2} \Delta v_{n-\frac{1}{2}} \Delta v_{n+\frac{1}{2}} \\
+ & \frac{1}{2}\left(f_{0-\frac{1}{2}}^{\prime} \Delta v_{0-\frac{1}{2}} \Delta v_{0+\frac{1}{2}}-f_{n+\frac{1}{2}}^{\prime} \Delta v_{n-\frac{1}{2}} \Delta v_{n+\frac{1}{2}}\right) \\
- & \frac{1}{2} a\left(\Delta v_{n-\frac{1}{2}}\right)^{2}+\frac{1}{2} a\left(\Delta v_{0+\frac{1}{2}}\right)^{2} \\
= & -\frac{a}{2}\left(\Delta v_{0+\frac{1}{2}}\right)^{2}+\frac{1}{2}\left(a+f_{0-\frac{1}{2}}^{\prime}\right) \Delta v_{0-\frac{1}{2}} \Delta v_{0+\frac{1}{2}}-\frac{a}{2}\left(\Delta v_{n-\frac{1}{2}}\right)^{2} \\
+ & \frac{1}{2}\left(a-f_{n+\frac{1}{2}}^{\prime}\right) \Delta v_{n-\frac{1}{2}} \Delta v_{n+\frac{1}{2}} \\
\leq & 0
\end{aligned}
$$

holds since $\Delta v_{0-\frac{1}{2}}$ and $\Delta v_{n+\frac{1}{2}}$ are less than or equal to zero. The desired result follows from the inequalities (3.15) and (3.19).

Finally, we deal with the case that $g^{E}(\cdot, \cdot)$ is the numerical flux of an $E$-scheme satisfying (2.11) and (2.12). It suffices to show that $P_{1} \leq 0$ and $P_{2} \leq 0$. We only give the proof of the former. The proof of the latter is similar and has been omitted.

Without loss of generality, let $s$ be the sonic point such that $v_{k} \leq s \leq v_{k+1}$ for some integer $k$ with $0 \leq k \leq n-1$. Then,

$$
\begin{gathered}
\left(\Delta f_{j+\frac{1}{2}}\right)^{+}=0 \quad \text { for } \quad 0 \leq j \leq k-1 \\
\left(\Delta f_{j+\frac{1}{2}}\right)^{+}=f_{j+\frac{1}{2}}^{\prime} \Delta v_{j+\frac{1}{2}} \quad \text { for } \quad n-1 \geq j \geq k+1 \\
\left(\Delta f_{j+\frac{1}{2}}\right)^{-}=0 \quad \text { for } \quad n-1 \geq j \geq k+1 ;
\end{gathered}
$$


and

$$
\left(\Delta f_{j+\frac{1}{2}}\right)^{-}=-f_{j+\frac{1}{2}}^{\prime} \Delta v_{j+\frac{1}{2}} \quad \text { for } \quad 0 \leq j \leq k-1 .
$$

Using these and the inequality (3.14), we deduce

$$
\begin{aligned}
P_{1} & =\sum_{j=0}^{n-1} \frac{\left(\Delta f_{j-\frac{1}{2}}\right)^{+}-\left(\Delta f_{j+\frac{1}{2}}\right)^{+}}{\left(\Delta f_{j-\frac{1}{2}}\right)^{+}+\left(\Delta f_{j+\frac{1}{2}}\right)^{+}}\left(\Delta f_{j+\frac{1}{2}}\right)^{+} \Delta v_{j+\frac{1}{2}} \\
& =-\left(\Delta f_{k+\frac{1}{2}}\right)^{+} \Delta v_{k+\frac{1}{2}} \\
& +\frac{\left(\Delta f_{k+\frac{1}{2}}\right)^{+}-f_{k+\frac{3}{2}}^{\prime} \Delta v_{k+\frac{3}{2}}}{\left(\Delta f_{k+\frac{1}{2}}\right)^{+}+f_{k+\frac{3}{2}}^{\prime} \Delta v_{k+\frac{3}{2}}} f_{k+\frac{3}{2}}^{\prime} \Delta v_{k+\frac{3}{2}} \Delta v_{k+\frac{3}{2}} \\
& +\sum_{j=k+2}^{n-1} \frac{f_{j-\frac{1}{2}}^{\prime} \Delta v_{j-\frac{1}{2}}-f_{j+\frac{1}{2}}^{\prime} \Delta v_{j+\frac{1}{2}}}{f_{j-\frac{1}{2}}^{\prime} \Delta v_{j-\frac{1}{2}}+f_{j+\frac{1}{2}}^{\prime} \Delta v_{j+\frac{1}{2}}}\left(f_{j+\frac{1}{2}}^{\prime} \Delta v_{j+\frac{1}{2}}\right) \Delta v_{j+\frac{1}{2}} \\
& \leq W_{1}+W_{2},
\end{aligned}
$$

where

$$
W_{1}:=-\left(\Delta f_{k+\frac{1}{2}}\right)^{+} \Delta v_{k+\frac{1}{2}}+\frac{\left(\Delta f_{k+\frac{1}{2}}\right)^{+}-f_{k+\frac{3}{2}}^{\prime} \Delta v_{k+\frac{3}{2}}}{2} \Delta v_{k+\frac{3}{2}}
$$

and

$$
W_{2}:=\frac{1}{2} \sum_{j=k+2}^{n-1}\left(f_{j-\frac{1}{2}}^{\prime} \Delta v_{j-\frac{1}{2}}-f_{j+\frac{1}{2}}^{\prime} \Delta v_{j+\frac{1}{2}}\right) \Delta v_{j+\frac{1}{2}}
$$

Next, for $k+2 \leq j \leq n-1$, the following inequality

$$
-\frac{1}{2} f_{j+\frac{1}{2}}^{\prime}\left(\Delta v_{j+\frac{1}{2}}\right)^{2} \leq-\frac{1}{4} f_{j-\frac{1}{2}}^{\prime}\left(\Delta v_{j+\frac{1}{2}}\right)^{2}-\frac{1}{4} f_{j+\frac{1}{2}}^{\prime}\left(\Delta v_{j+\frac{1}{2}}\right)^{2}
$$

enables us to obtain an upper bound of $W_{2}$

$$
\begin{aligned}
W_{2} & =\frac{1}{2} \sum_{j=k+2}^{n-1}\left(f_{j-\frac{1}{2}}^{\prime} \Delta v_{j-\frac{1}{2}}-f_{j+\frac{1}{2}}^{\prime} \Delta v_{j+\frac{1}{2}}\right) \Delta v_{j+\frac{1}{2}} \\
& \leq \frac{1}{4} f_{k+\frac{3}{2}}^{\prime}\left(\Delta v_{k+\frac{3}{2}}\right)^{2}-\frac{1}{4} f_{n-\frac{1}{2}}^{\prime}\left(\Delta v_{n-\frac{1}{2}}\right)^{2} \\
& -\frac{1}{4} \sum_{j=k+2}^{n-1} f_{j-\frac{1}{2}}^{\prime}\left(\Delta v_{j-\frac{1}{2}}-\Delta v_{j+\frac{1}{2}}\right)^{2} \\
& \leq \frac{1}{4} f_{k+\frac{3}{2}}^{\prime}\left(\Delta v_{k+\frac{3}{2}}\right)^{2}
\end{aligned}
$$


and therefore, by letting $\Delta v_{k+\frac{3}{2}}=c \Delta v_{k+\frac{1}{2}}$

$$
\begin{aligned}
P_{1} & \leq W_{1}+W_{2} \\
& \leq-\left(\Delta f_{k+\frac{1}{2}}\right)^{+} \Delta v_{k+\frac{1}{2}}+\frac{1}{4} f_{k+\frac{3}{2}}^{\prime}\left(\Delta v_{k+\frac{3}{2}}\right)^{2} \\
& +\frac{\left(\Delta f_{k+\frac{1}{2}}\right)^{+}-f_{k+\frac{3}{2}}^{\prime} \Delta v_{k+\frac{3}{2}}}{2} \Delta v_{k+\frac{3}{2}} \\
& =-\left(D f_{k+\frac{1}{2}}\right)^{+}\left(\Delta v_{k+\frac{1}{2}}\right)^{2}+\frac{1}{2}\left(D f_{k+\frac{1}{2}}\right)^{+} \Delta v_{k+\frac{1}{2}} \Delta v_{k+\frac{3}{2}} \\
& -\frac{1}{4} f_{k+\frac{3}{2}}^{\prime}\left(\Delta v_{k+\frac{3}{2}}\right)^{2} \\
& =Q(c)\left(\Delta v_{k+\frac{1}{2}}\right)^{2},
\end{aligned}
$$

where

$$
Q(c)=-\left(D f_{k+\frac{1}{2}}\right)^{+}+\frac{c}{2}\left(D f_{k+\frac{1}{2}}\right)^{+}-\frac{c^{2}}{4} f_{k+\frac{3}{2}}^{\prime} .
$$

It is easy to see that $Q(c)$ reaches its maximum at $c_{0}=\frac{\left(D f_{k+\frac{1}{2}}\right)^{+}}{f_{k+\frac{3}{2}}^{\prime}}$. Thus to show $P_{1} \leq 0$, it suffices to show $Q\left(c_{0}\right) \leq 0$.

Note that

$$
\begin{aligned}
& Q\left(c_{0}\right)=\frac{\left(D f_{k+\frac{1}{2}}\right)^{+}}{4 f_{k+\frac{3}{2}}^{\prime}}\left[\left(D f_{k+\frac{1}{2}}\right)^{+}-4 f_{k+\frac{3}{2}}^{\prime}\right] \\
= & \frac{\left(D f_{k+\frac{1}{2}}\right)^{+}}{4 f_{k+\frac{3}{2}}^{\prime}}\left[\left(D f_{k+\frac{1}{2}}\right)^{+}-4 f^{\prime}\left(\xi_{k+1}\right)\right],
\end{aligned}
$$

where $\xi_{k+1}$ is in between $v_{k+1}$ and $v_{k+2}$. Next the convexity of $f$ implies that $f^{\prime}\left(v_{k+1}\right) \leq f^{\prime}\left(\xi_{k+1}\right)$. Thus, by $(2.11), f\left(v_{k+1}\right)-g^{E}\left(v_{k}, v_{k+1}\right) \leq 4 f^{\prime}\left(v_{k+1}\right) \Delta v_{k+\frac{1}{2}} \leq$ $4 f^{\prime}\left(\xi_{k+1}\right) \Delta v_{k+\frac{1}{2}}$ ensures that $Q\left(c_{o}\right) \leq 0$ as desired. The proof is completed.

\section{REFERENCES}

[1] S. Chakravarthy and S. Osher, High resolution applications of the Osher upwind scheme for the Euler equations, AIAA paper presented at 6th CFD conference 1983.

[2] P. Colella And P. Woodward, The piecewise-parabolic method (PPM) for gas-dynamical simulations, J. Comput. Phys., 54 (1984), pp. 174-201.

[3] R. J. DiPerna, Convergence of approximate solutions to conservation laws, Arch. Rational Mech. Aual., 82 (1983), pp. 27-70.

[4] B. Engquist AND S. Osher, Stable and entropy satisfying approximations for transonic flow calculations, Math. Comp., 34 (1980), pp. 45-75.

[5] S.K. Godunov, Finite-difference method for numerical computation of discontinuous solutions of the equations of fluid dynamics, Mat. Sbornik, 47 (1959), pp. 271-306.

[6] A. Harten, B. Engquist, S. Osher, and S. Chakravarthy, Uniformly high order accurate essentially nonoscillatory schemes, III, J. Comput. Phys., 71 (1987), p. 231.

[7] A. Harten, J. M. Hyman and P. D. Lax, On finite-difference approximations and entropy conditions for shocks, Comm. Pure and Appl. Math., 29 (1976), pp. 297-322.

[8] A. HaRTen AND S. OsheR, Uniformly high-order accurate nonoscillatory schemes. I, SIAM J. Num. Anal., 24 (1987), pp. 279-309

[9] JiAng, G.-S., AND SHU, C.-W, Efficient implementation of weighted ENO schemes, J. Comput. Phys., 126:1 (1996), pp. 202-228. 
[10] S. N. KRUZKov, first order quasilinear equations in several independent variables, Math. USSR Sb., 47 (1970), pp. 217-243.

[11] P. Lax And B. Wendroff, Systems of conservation laws,Comm. Pure Appl. Math., 13 (1960), pp. 217-237.

[12] P. LAx, Hyperbolic Systems of Conservation Laws and the Mathematical Theory of Shock Waves, SIAM Regional Conference Series in Applied Mathematics, 11 (1972).

[13] Liu, X.-D., Osher, S., And Chan, T., Weighed essentially non-oscillatory schemes, J. Comput. Phys., 115 (1994), pp. 200-212.

[14] S. OSHER, Riemann solvers, the entropy condition and difference approximations, SIAM J. Numer. Anal., 21 (1984), pp. 217-235.

[15] S. Osher ANd S. Chakravarthy, High resolution schemes and entropy condition, SIAM J. Numer. Anal., 21 (1984), pp. 955-984.

[16] S. OSHER AND E. TADMOR, On the convergence of difference schemes to scalar conservation laws, Math. Comp., 50 (1988), pp. 19-51.

[17] P. L. Roe, Some contributions to the modeling of discontinuous flows, Lect. Notes Appl. Math., 22 (1985), pp. 163-193.

[18] Shu, C.-W., AND Osher, S., Efficient implementation of essentially non-oscillatory shock capturing schemes, J. Comp. Phys., 83 (1989), p. 32.

[19] P. K. Sweby, High resolution schemes using flux limiters for hyperbolic conservation laws, SIAM J. Numer. Anal., 21 (1984), pp. 995-1011.

[20] E. TADMOR, Convenient total variation diminishing conditions for nonlinear difference schemes, SIAM J. Numer. Anal., 25 (1988), pp. 1002-1014.

[21] B. VAN LEER, Towards the ultimate conservative difference scheme II. Monotonicity and conservation combined in a second order scheme, J. Comput. Phys., 14 (1974), pp. 361-370.

[22] A. Vol'Pert, The space BV and quasi-linear equations, Math. USSR Sb, 73 (1967), pp. 255302.

[23] H. YAng, On Wavewise Entropy Inequalities for High-Resolution Schemes.I:The Semi-discrete case, Math. Comp., 65 (1996), pp. 45-67.

[24] H. YANG, On Wavewise Entropy Inequalities for High Resolution Schemes II: Fully discrete MUSCL Schemes with Exact Evolution In Small time, SIAM. J. Numer. Anal., 36:1 (1999), pp. 1-31.

[25] H. YANG AND N. JiAng, On Wavewise Entropy Inequalities for High-Resolution Schemes with source terms I:The Semi-discrete case, Methods and Applications of Analysis, 10:4 (2003), pp. $487-512$. 
